In-situ Evaluation of Laminates with Gaps and Overlaps Uncertainties in Modelling and Analysis

Falk Heinecke, Tobias Wille

German Aerospace Center (DLR)

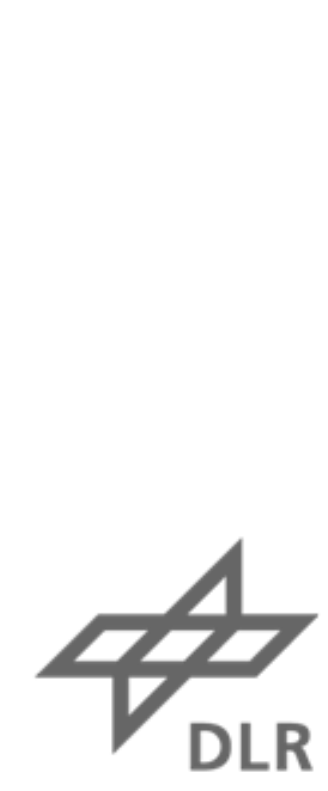




\section{Preparing a Tiramisu}

- Ingredients determine the quality of the Tiramisu

- How to check whether the eggs are still consumable?
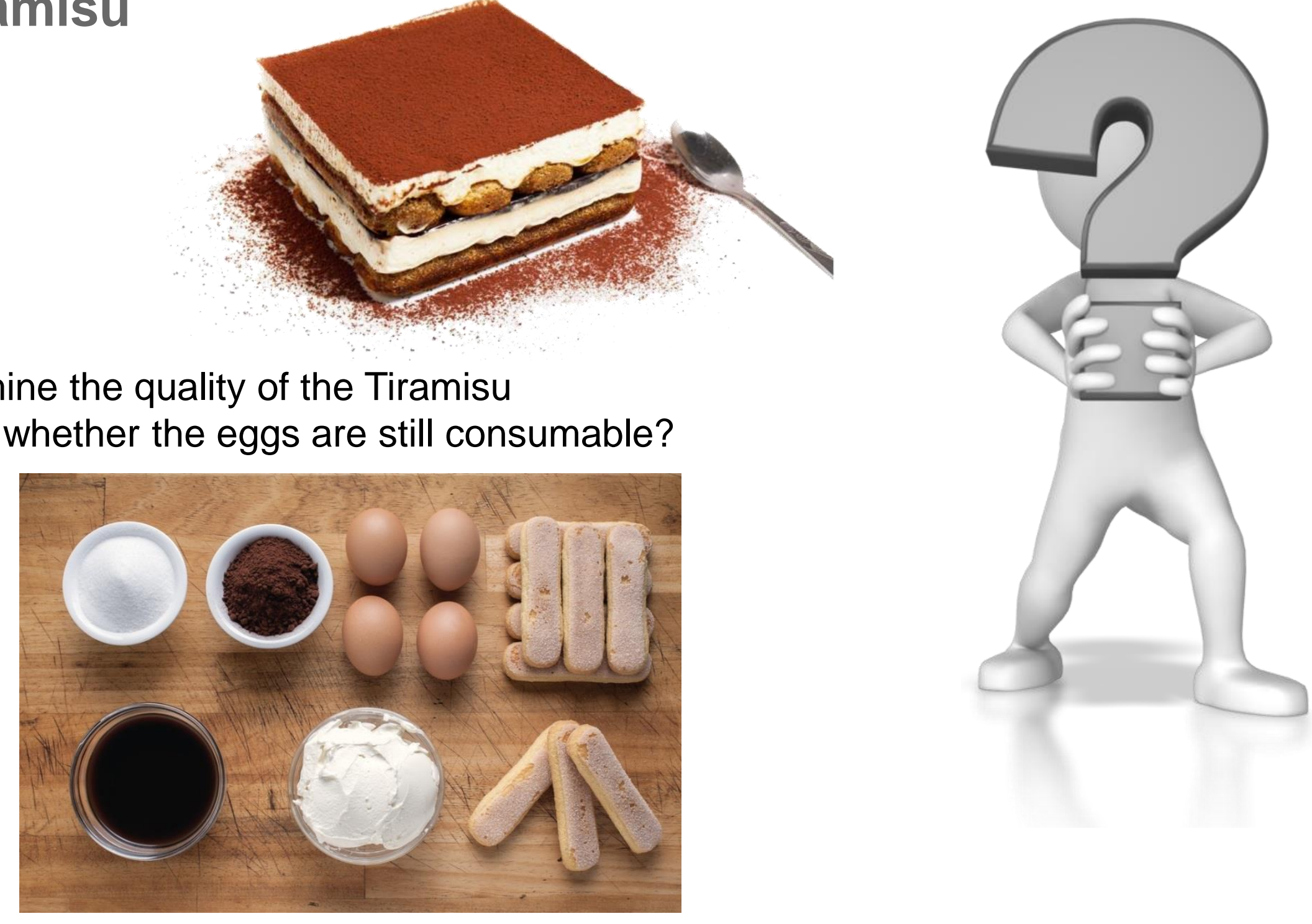


\section{Manufacturing a laminate using Automated Fibre Placement (AFP)}

- Typical manufacturing induced deviations:

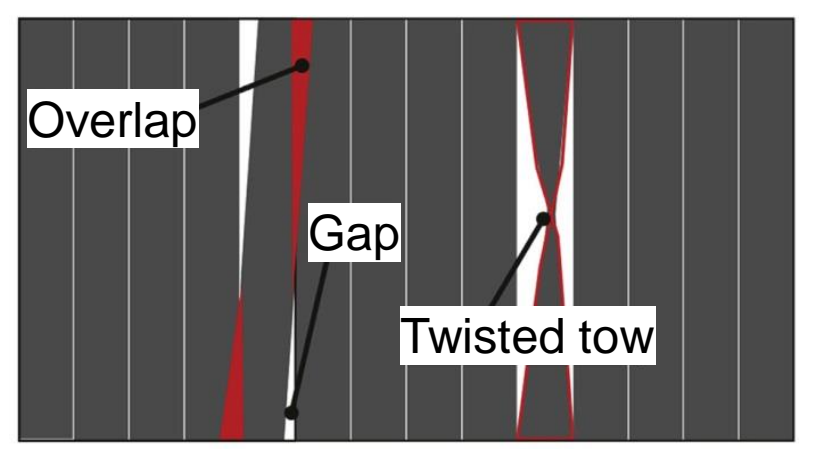

Source:[Denkena2016]

- AFP cycle time distribution:

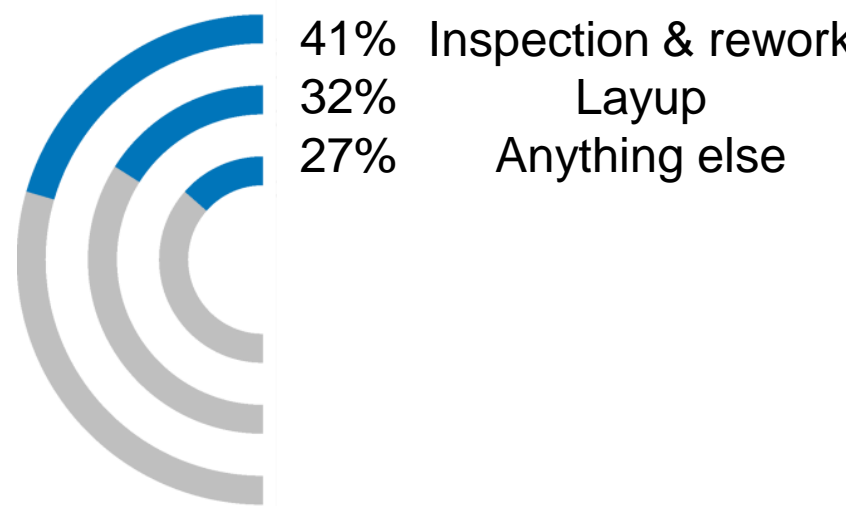

Source: according to [Rudberg2014]

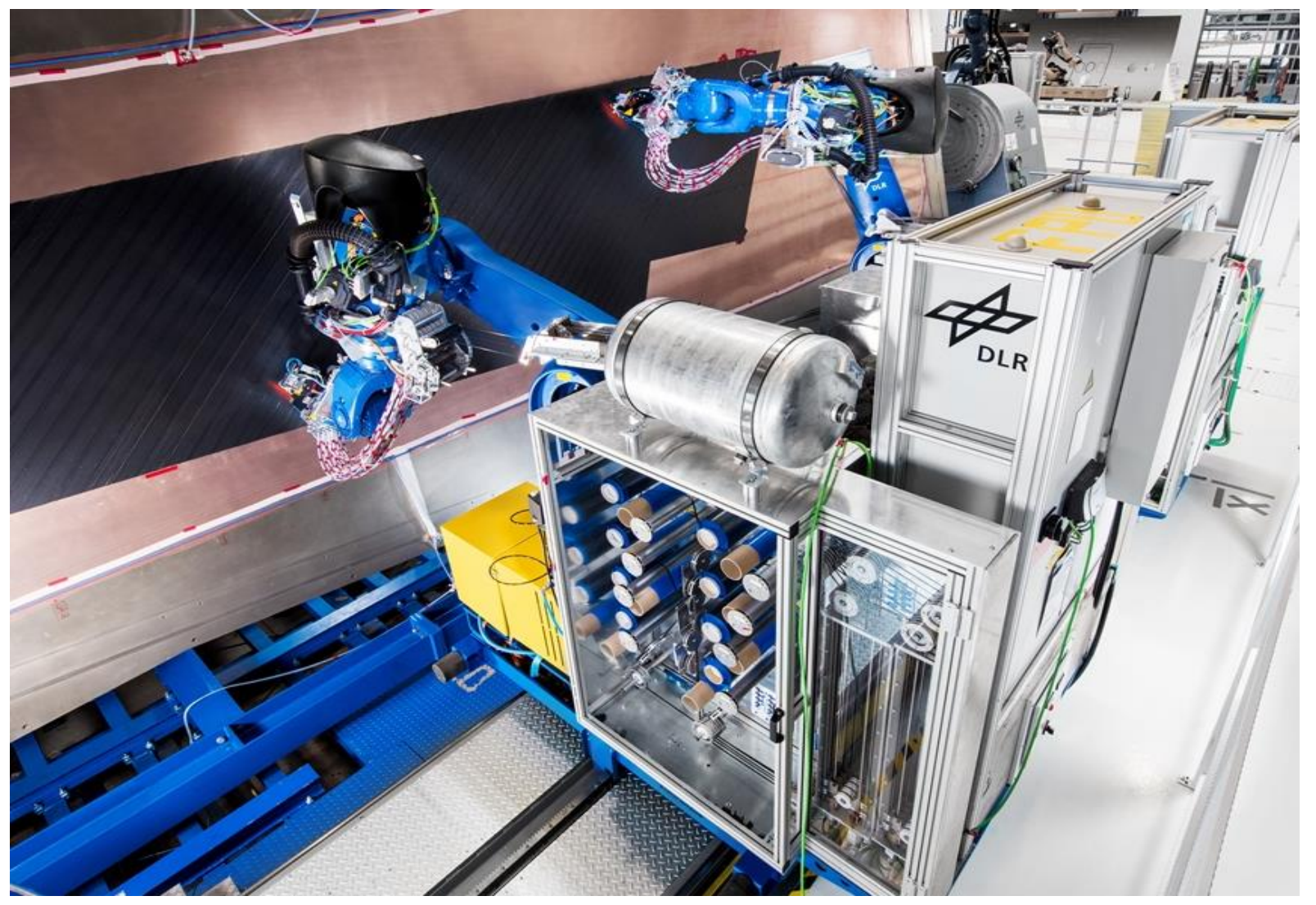




\section{In-situ evaluation of laminates - The enablers}

\section{Online monitoring}

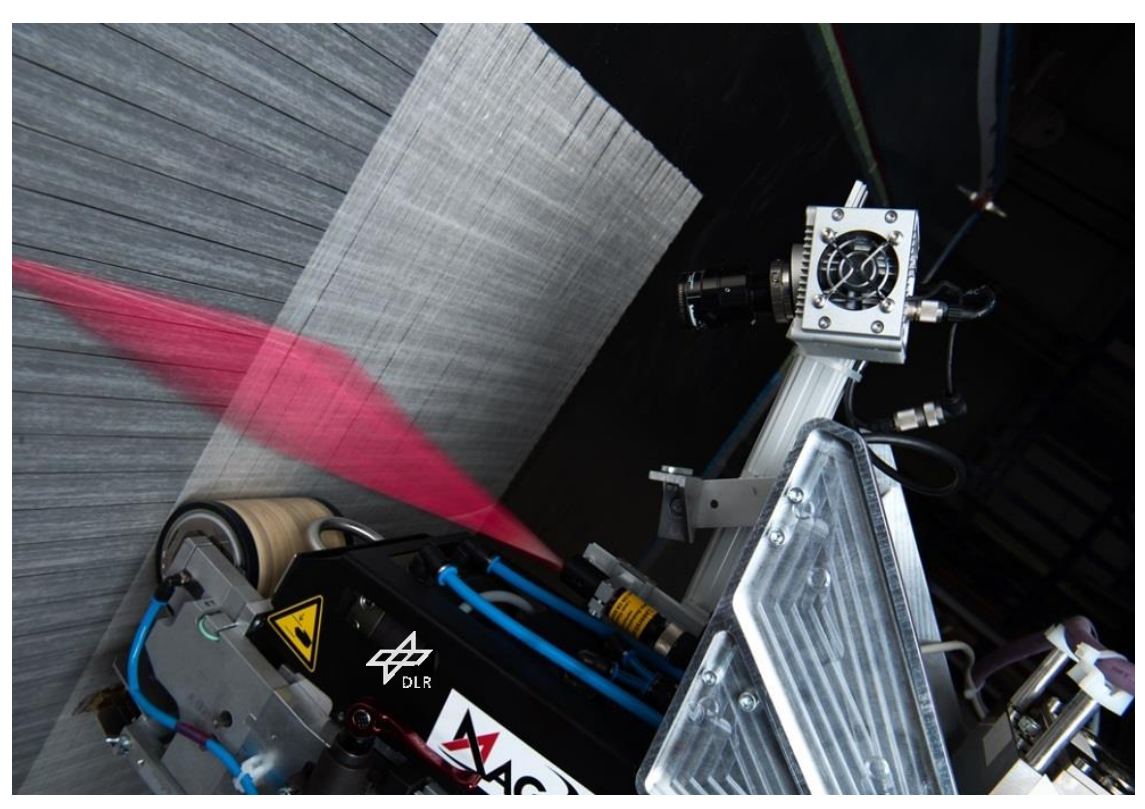

- Realtime capable measurement systems and algorithms

- Instead of manual inspection

\section{In-Situ evaluation and assessment tools}

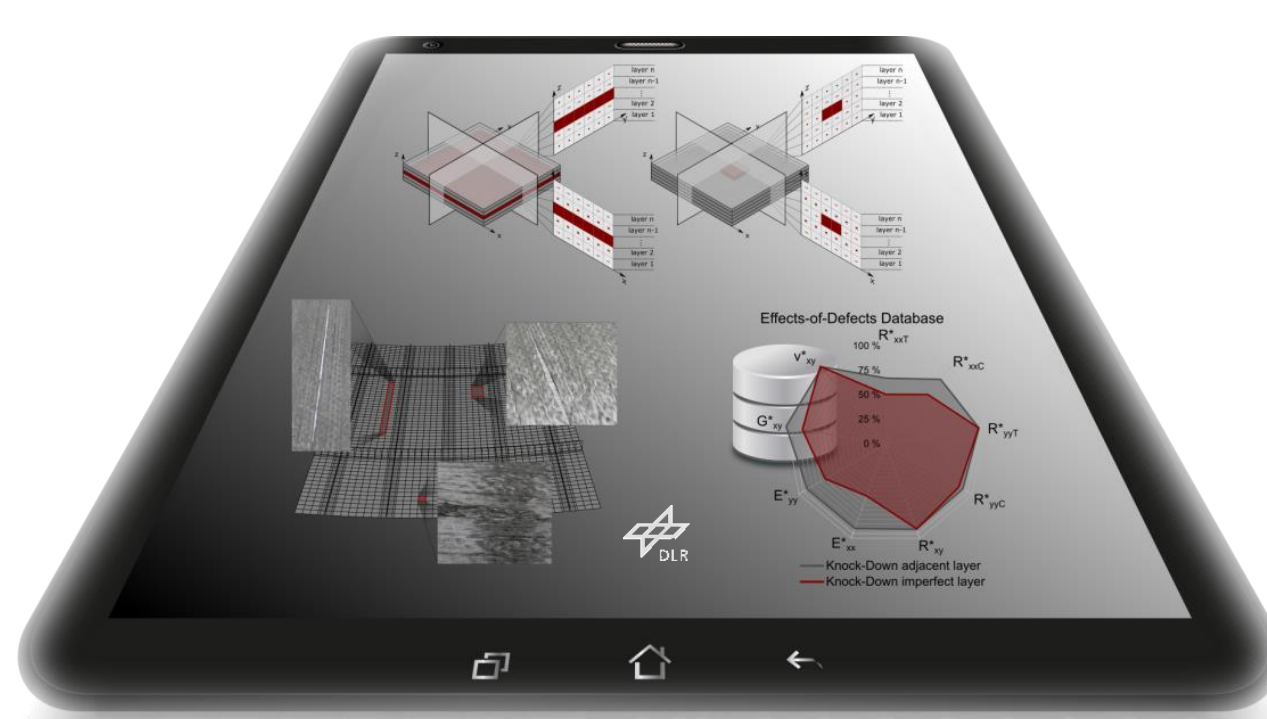

- Realtime capable evaluation systems and algorithms

- Instead of conservative composite engineering requirements/ allowables

Heinecke et al.: In-situ structural evaluation during the fibre deposition process of composite manufacturing, CEAS Aeronautical Journal, 9:123-133, 2018 


\section{In-situ evaluation of laminates - Uncertainty factors}

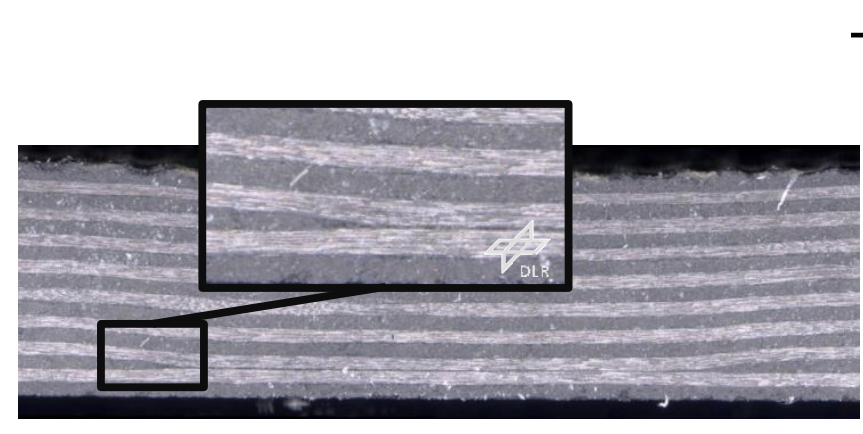

Transfer from

Reality to

Model

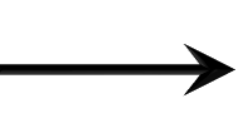

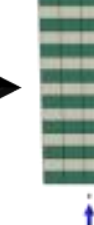

- From Reality to Phenomenology:

Transfer from Model

to KDF
Effects-of-Defects Database

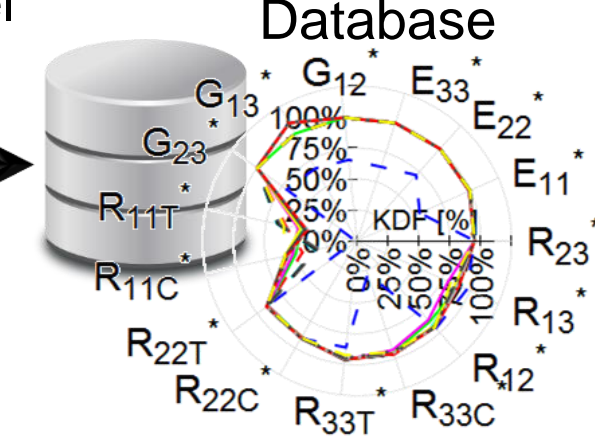

- Which simplifications are valid?

- Which assumptions are valid?

- From Phenomenology to Modelling:

- Which boundary conditions to use in model?

- Which failure criterion to use in model?

- How to transfer the high-fidelity result into a simple Knock-Down-Factor (KDF)? 


\section{Transferring „real“ world into model world - From Reality to Phenomenology}

-What happens after fibre deposition?

- Influence of subsequent manufacturing process steps [Hassan2017,Belnoue2017]

-Which geometry characteristics are relevant?

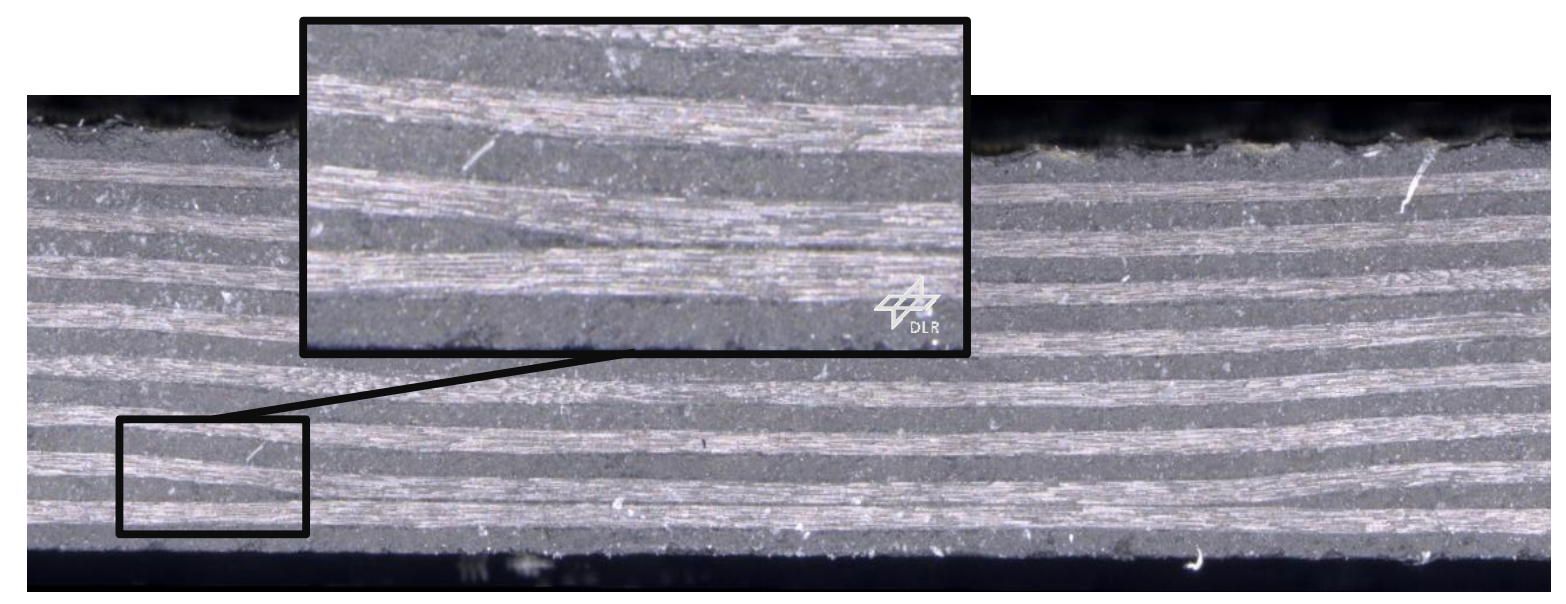

- Influence of modelling approaches (e.g. Defect Layer Method [Fayazbakhsh2013])
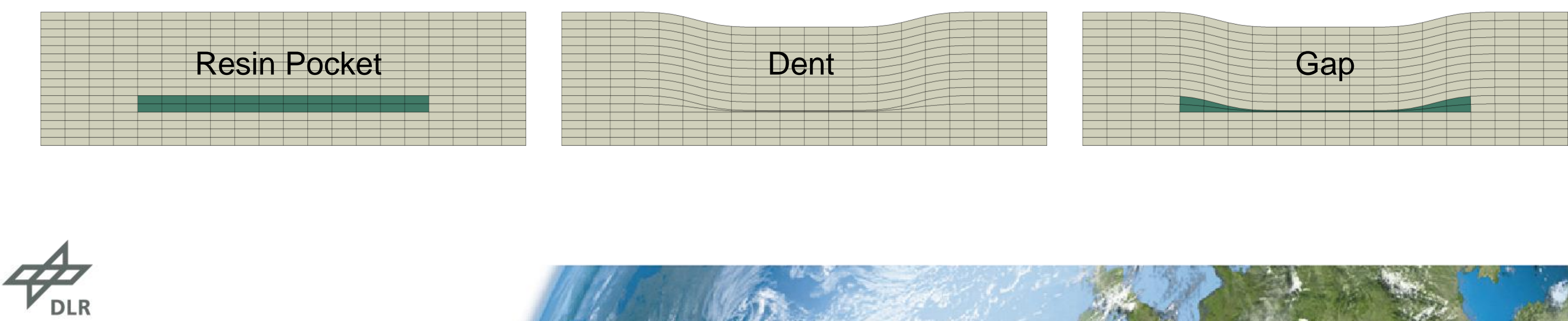


\section{Transferring „real“ world into model world - From Phenomenology to Modelling}

\section{Stiffness Homogenization}

- Classical boundary value problem

- Volume-averaging stresses and strains

\section{Strength Homogenization}

- Material effort (M) calculated from failure criterion

- Evaluation of local stresses (no averaging)
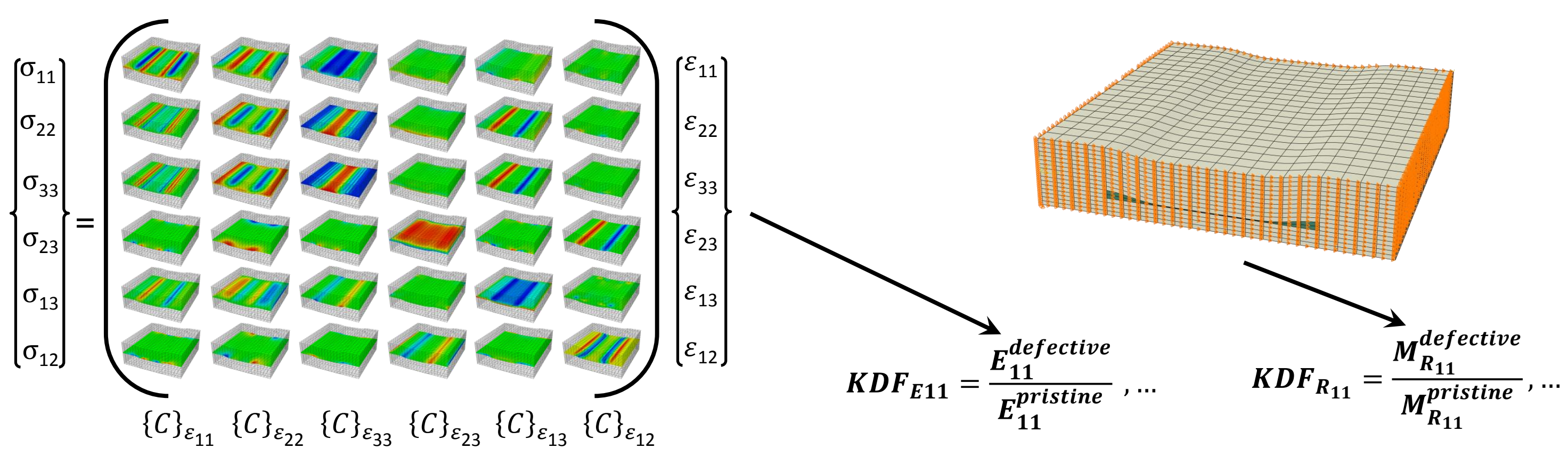


\section{Stiffness Homogenization - From Phenomenology to Modelling}

- Homogeneous displacement BC's ("plane-remains-plane" [Xia2006]) used for numerical homogenization (FEModel)

\begin{tabular}{cccccccccc}
\hline $\begin{array}{l}\text { Engineering } \\
\text { Constants }\end{array}$ & \multicolumn{1}{l}{ Resin Pocket (KDF) } & Dent (KDF) & & Gap (KDF) \\
\hline & $\begin{array}{c}\text { Reuss- } \\
\text { Model }\end{array}$ & $\begin{array}{l}\text { FE- } \\
\text { Model }\end{array}$ & $\begin{array}{c}\text { Voigt- } \\
\text { Model }\end{array}$ & $\begin{array}{c}\text { Reuss- } \\
\text { Model }\end{array}$ & $\begin{array}{c}\text { FE- } \\
\text { Model }\end{array}$ & $\begin{array}{c}\text { Voigt- } \\
\text { Model }\end{array}$ & $\begin{array}{c}\text { Reuss- } \\
\text { Model }\end{array}$ & $\begin{array}{c}\text { FE- } \\
\text { Model }\end{array}$ & $\begin{array}{c}\text { Voigt- } \\
\text { Model }\end{array}$ \\
\hline$E_{x}$ & $23 \%$ & $98 \%$ & $98 \%$ & $27 \%$ & $96 \%$ & $102 \%$ & $26 \%$ & $95 \%$ & $101 \%$ \\
$E_{y}$ & $21 \%$ & $81 \%$ & $81 \%$ & $24 \%$ & $87 \%$ & $92 \%$ & $23 \%$ & $80 \%$ & $85 \%$ \\
$E_{z}$ & $74 \%$ & $96 \%$ & $97 \%$ & $79 \%$ & $95 \%$ & $100 \%$ & $77 \%$ & $93 \%$ & $99 \%$ \\
$G_{x y}$ & $25 \%$ & $98 \%$ & $98 \%$ & $31 \%$ & $98 \%$ & $104 \%$ & $28 \%$ & $98 \%$ & $103 \%$ \\
$G_{x z}$ & $92 \%$ & $95 \%$ & $111 \%$ & $102 \%$ & $102 \%$ & $122 \%$ & $99 \%$ & $99 \%$ & $122 \%$ \\
$G_{y z}$ & $88 \%$ & $92 \%$ & $105 \%$ & $98 \%$ & $96 \%$ & $113 \%$ & $94 \%$ & $93 \%$ & $110 \%$ \\
$v_{x y}$ & $50 \%$ & $120 \%$ & $120 \%$ & $30 \%$ & $112 \%$ & $104 \%$ & $38 \%$ & $119 \%$ & $119 \%$ \\
$v_{x z}$ & $122 \%$ & $92 \%$ & $92 \%$ & $129 \%$ & $99 \%$ & $122 \%$ & $126 \%$ & $96 \%$ & $98 \%$ \\
$v_{y z}$ & $124 \%$ & $100 \%$ & $100 \%$ & $130 \%$ & $99 \%$ & $113 \%$ & $128 \%$ & $99 \%$ & $99 \%$ \\
\hline
\end{tabular}

Voigt-Model (iso-strain)

$$
\left[C_{l m}\right]^{V}=\sum_{i=1}^{n} v_{i}\left[C_{l m}\right]^{i}
$$

Reuss-Model (iso-stress)

$\left[C_{l m}\right]^{R}=\left[\sum_{i=1}^{n} v_{i}\left[S_{l m}\right]^{i}\right]^{-1}$ 


\section{Stiffness Homogenization - The essence}

- Negligible influence from modelling approach...

-...Depending on modelling approach - Maximum 8\% Variation in Results

- Homogeneous displacement boundary conditions provide "iso-strain-like" results...

- ....in contrast to periodic displacement boundary conditions or stress boundary conditions [Glüge2013] 


\section{Homogenization of Strength - From Phenomenology to Modelling}

- Strength properties: Pre-preg Hexply IM7/8552

- Uniaxial tensile stress - Limit Stress versus Puck 3D

- Failure mode switches - I: Fibre fracture; II \& III: Interfibre fracture
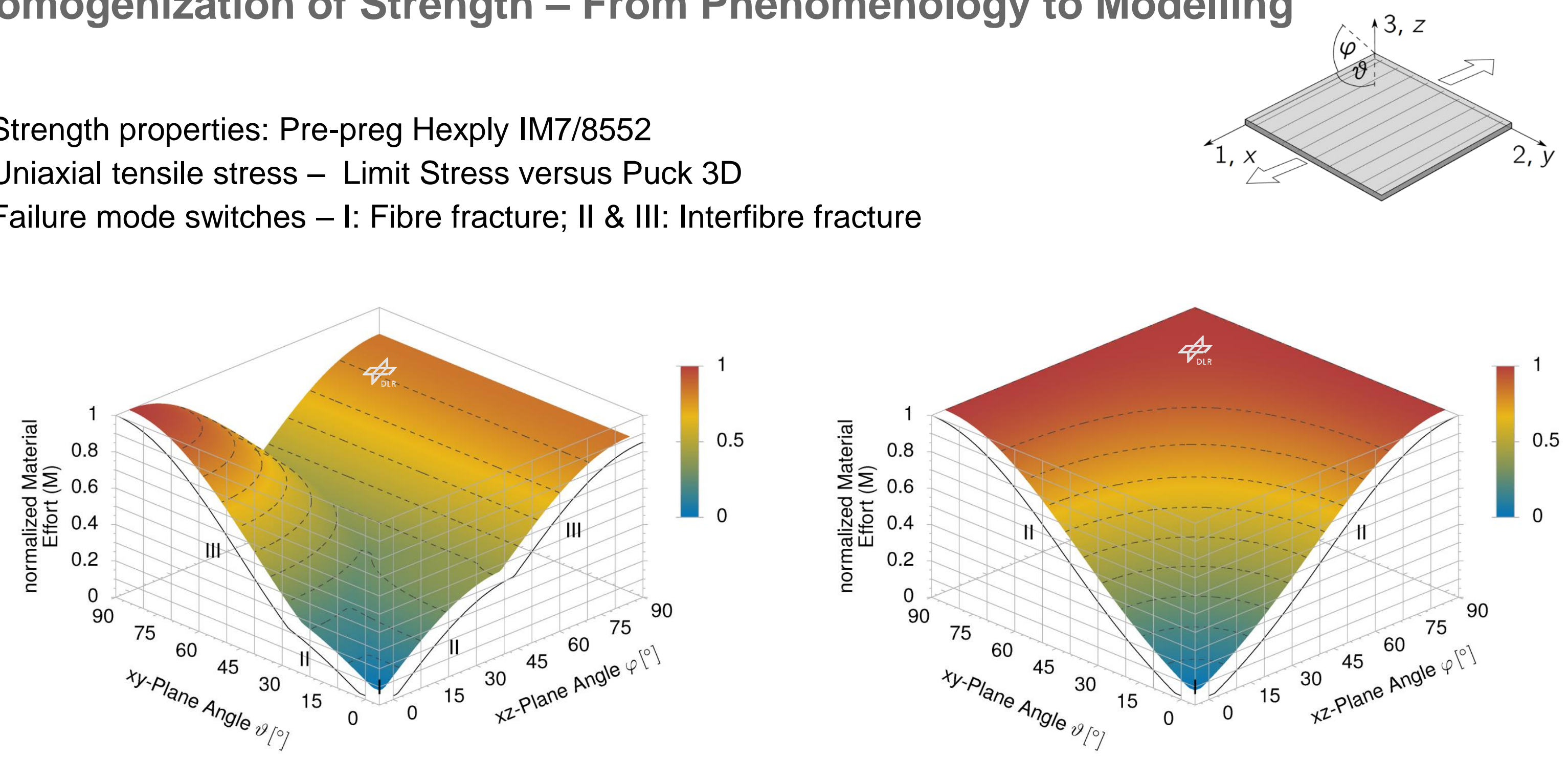


\section{Homogenization of Strength - From Phenomenology to Modelling}

- Strength properties: Pre-preg Hexply IM7/8552

- Uniaxial compression stress - Limit Stress versus Puck 3D

- Failure mode switches - I: Fibre fracture; II \& III: Interfibre fracture
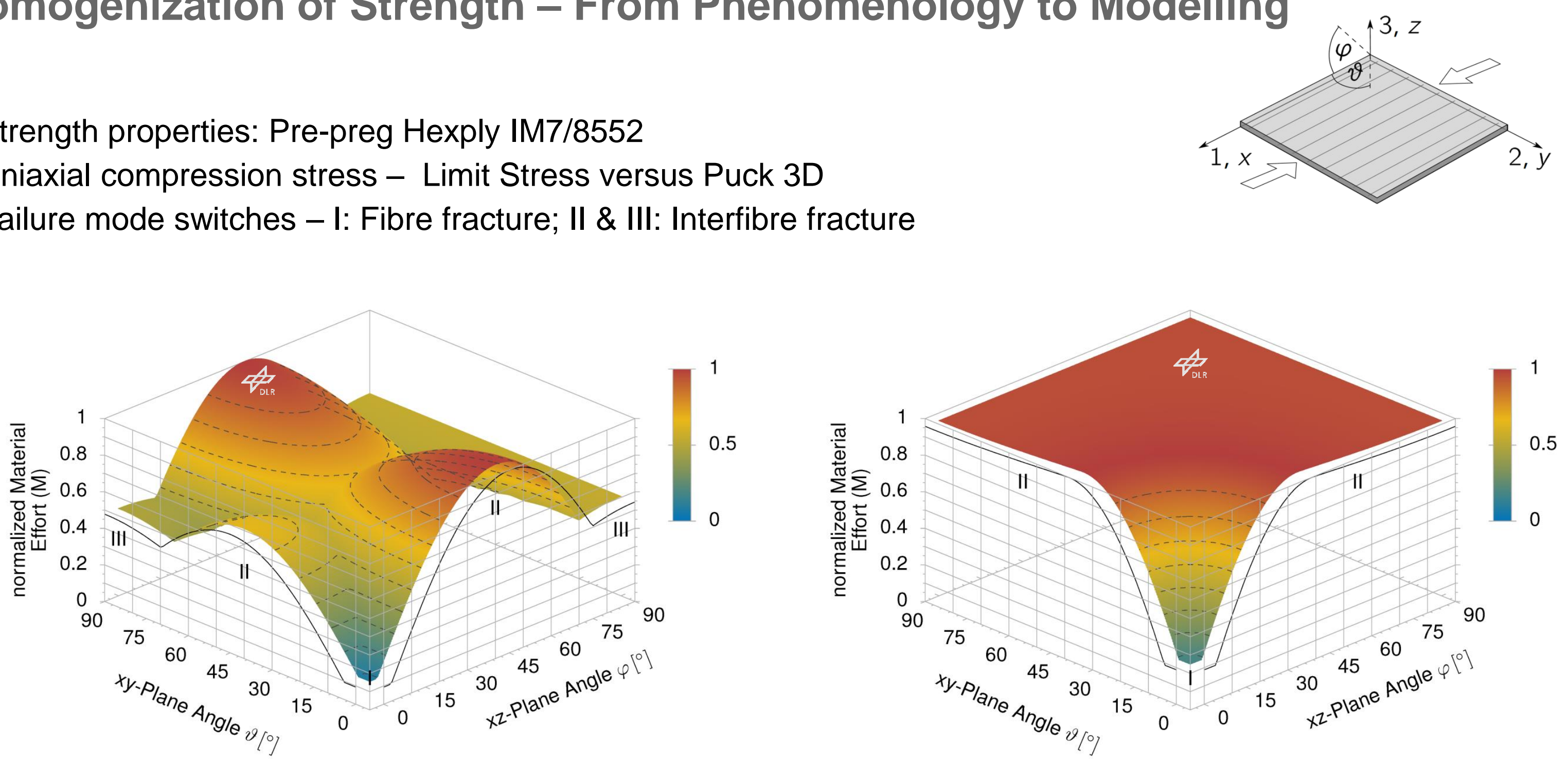


\section{Homogenization of Strength - Laminate failure}

- Linear static analysis combined with First-Ply-Failure approach used to compare pristine failure versus defective failure

\begin{tabular}{|c|c|c|c|c|c|c|c|c|c|c|c|c|}
\hline \multirow[t]{3}{*}{ Strength } & \multicolumn{4}{|c|}{ Resin Pocket (KDF) } & \multicolumn{4}{|c|}{ Dent (KDF) } & \multicolumn{4}{|c|}{ Gap (KDF) } \\
\hline & \multicolumn{2}{|c|}{ Homog. BC's } & \multicolumn{2}{|c|}{ In-Plane BC's } & \multicolumn{2}{|c|}{ Homog. BC's } & \multicolumn{2}{|c|}{ In-Plane BC's } & \multicolumn{2}{|c|}{ Homog. BC's } & \multicolumn{2}{|c|}{ In-Plane BC's } \\
\hline & $\begin{array}{l}\text { Limit } \\
\text { Stress }\end{array}$ & Puck & $\begin{array}{l}\text { Limit } \\
\text { Stress }\end{array}$ & Puck & $\begin{array}{l}\text { Limit } \\
\text { Stress }\end{array}$ & Puck & $\begin{array}{l}\text { Limit } \\
\text { Stress }\end{array}$ & Puck & $\begin{array}{l}\text { Limit } \\
\text { Stress }\end{array}$ & Puck & $\begin{array}{l}\text { Limit } \\
\text { Stress }\end{array}$ & Puck \\
\hline$R_{11 T}$ & $93 \%$ & $98 \%$ & $91 \%$ & $99 \%$ & $84 \%$ & $84 \%$ & $58 \%$ & $50 \%$ & $95 \%$ & $84 \%$ & $94 \%$ & $52 \%$ \\
\hline$R_{11 C}$ & $99 \%$ & $99 \%$ & $94 \%$ & $76 \%$ & $92 \%$ & $92 \%$ & $46 \%$ & $49 \%$ & $91 \%$ & $91 \%$ & $45 \%$ & $48 \%$ \\
\hline$R_{22 T}$ & $83 \%$ & $83 \%$ & $75 \%$ & $78 \%$ & $86 \%$ & $86 \%$ & $79 \%$ & $84 \%$ & $81 \%$ & $81 \%$ & $70 \%$ & $77 \%$ \\
\hline$R_{22 C}$ & $83 \%$ & $92 \%$ & $79 \%$ & $78 \%$ & $88 \%$ & $88 \%$ & $76 \%$ & $76 \%$ & $82 \%$ & $82 \%$ & $79 \%$ & $79 \%$ \\
\hline$R_{33 T}$ & $92 \%$ & $92 \%$ & $92 \%$ & $92 \%$ & $93 \%$ & $93 \%$ & $93 \%$ & $93 \%$ & $92 \%$ & $92 \%$ & $92 \%$ & $92 \%$ \\
\hline$R_{33 C}$ & $92 \%$ & $70 \%$ & $92 \%$ & $70 \%$ & $93 \%$ & $91 \%$ & $93 \%$ & $76 \%$ & $92 \%$ & $63 \%$ & $92 \%$ & $63 \%$ \\
\hline$R_{12}$ & $90 \%$ & $90 \%$ & $90 \%$ & $90 \%$ & $97 \%$ & $97 \%$ & $94 \%$ & $87 \%$ & $88 \%$ & $87 \%$ & $88 \%$ & $85 \%$ \\
\hline$R_{13}$ & $85 \%$ & $84 \%$ & $80 \%$ & $78 \%$ & $70 \%$ & $70 \%$ & $74 \%$ & $73 \%$ & $90 \%$ & $86 \%$ & $87 \%$ & $82 \%$ \\
\hline$R_{23}$ & $84 \%$ & $83 \%$ & $83 \%$ & $82 \%$ & $94 \%$ & $89 \%$ & $94 \%$ & $89 \%$ & $89 \%$ & $85 \%$ & $88 \%$ & $88 \%$ \\
\hline
\end{tabular}




\section{Strength Homogenization - The essence}

- Depending on combination of modelling approach and boundary conditions...

- ...Significant influence from failure criterion - Up to $20 \%$ Variation in Results

-...Significant influence from boundary conditions - Over 30\% Variation in Results

- Significant influence from modelling approach

- Fibre undulation is failure driving [Garnich2005, Hsaio1996] 


\section{Key messages}

- Reasonable choice of a model:

- Accuracy versus complexity - Not because it is always done this way

- Don't struggle with uncertainties

- They are everyway - Try to identify their sources and influence

- In-situ evaluation of laminates means also effectiveness:

- The adequate model to accomplish the purpose

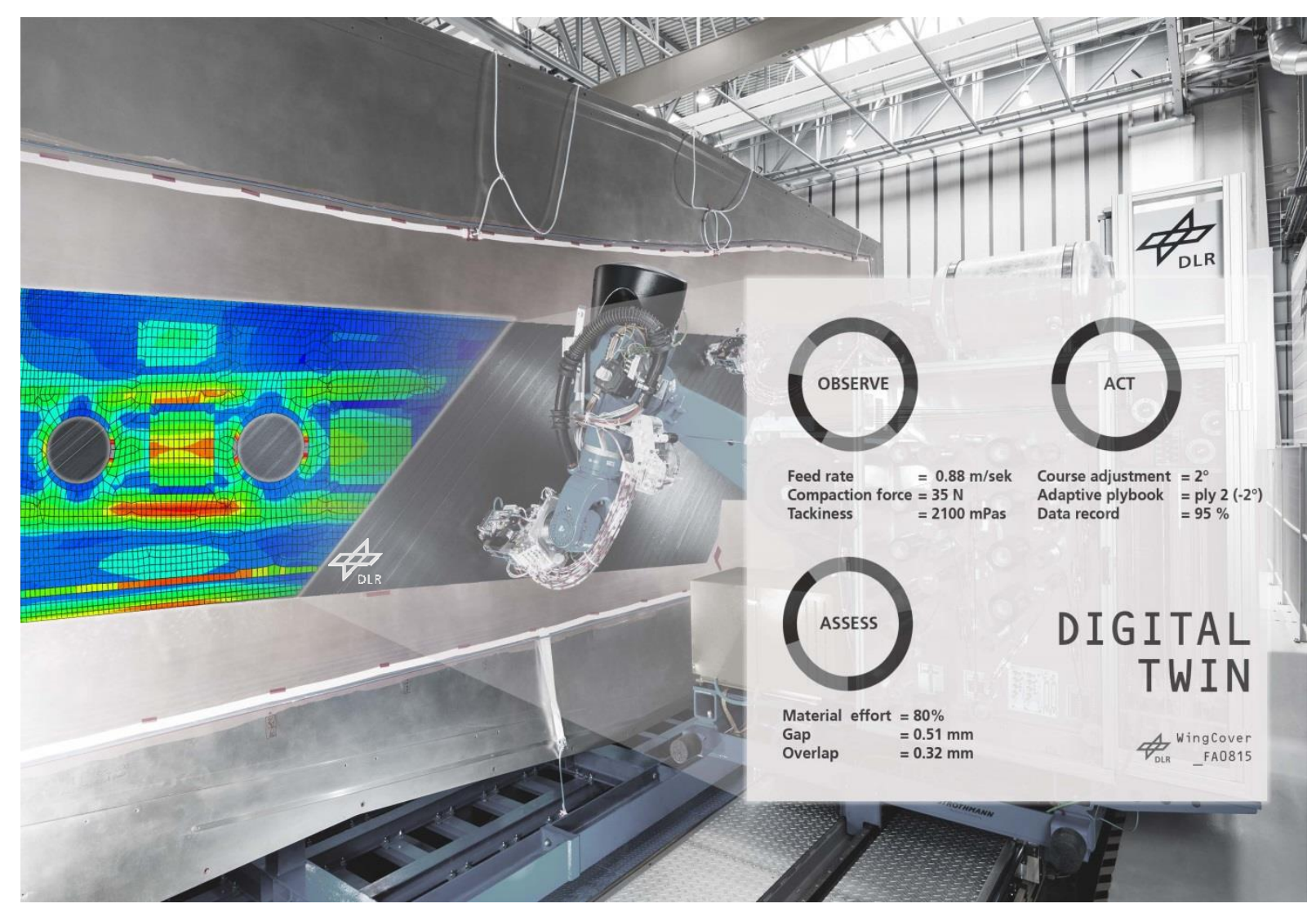




\section{Questions and comments are very welcome!}

"What makes modelling and scientific inquiry in general so painful is uncertainty. Uncertainty is not an accident of the scientific method, but its substance."

A. Saltelli

Contact:

Falk Heinecke

Phone: +49 531-295-2312

Email: falk.heinecke@dlr.de 


\section{References}

- [Heinecke2018]: F. Heinecke and T. Wille, "In-situ structural evaluation during the fibre deposition process of composite manufacturing," CEAS Aeronaut. J., 2018.

- [Hassan2017]: M. H. Hassan, A. R. Othman, and S. Kamaruddin, "A review on the manufacturing defects of complex-shaped laminate in aircraft composite structures," Int. J. Adv. Manuf. Technol., vol. 91, no. 9-12, pp. 4081-4094, 2017.

- [Belnoue2017]: J. P. H. Belnoue, T. Mesogitis, O. J. Nixon-Pearson, J. Kratz, D. S. Ivanov, I. K. Partridge, K. D. Potter, and S. R. Hallett, "Understanding and predicting defect formation in automated fibre placement pre-preg laminates," Compos. Part A Appl. Sci. Manuf., vol. 102, pp. 196-206, 2017.

- [Denkena2016]: B. Denkena, C. Schmidt, K. Völtzer, and T. Hocke, "Thermographic online monitoring system for Automated Fiber Placement processes," Compos. Part B Eng., vol. 97, pp. 239-243, 2016.

- [Rudberg2014]: T. Rudberg, J. Nielson, M. Henscheid, and J. Cemenska, "Improving AFP Cell Performance," SAE Int. J. Aerosp., vol. 7, no. 2, pp. 2014-01-2272, 2014.

- [Glüge2013]: R. Glüge, "Generalized boundary conditions on representative volume elements and their use in determining the effective material properties," PAMM, vol. 13, no. 1, pp. 251-252, Dec. 2013.

- [Fayazbakhsh2013]: K. Fayazbakhsh, M. A. Nik, D. Pasini, and L. Lessard, "Defect layer method to capture effect of gaps and overlaps in variable stiffness laminates made by Automated Fiber Placement," Compos. Struct., vol. 97, pp. 245-251, Mar. 2013.

- [Xia2006]: Z. Xia, C. Zhou, Q. Yong, and X. Wang, "On selection of repeated unit cell model and application of unified periodic boundary conditions in micro-mechanical analysis of composites," Int. J. Solids Struct., vol. 43, no. 2, pp. 266-278, Jan. 2006.

- [Garnich2005]: M. R. Garnich and G. Karami, "Localized Fiber Waviness and Implications for Failure in Unidirectional Composites," J. Compos. Mater., vol. 39, no. 14, pp. 1225-1245, Jul. 2005.

- [Hsia01996]: H. M. Hsiao and I. M. Daniel, "Effect of fiber waviness on stiffness and strength reduction of unidirectional composites under compressive loading," Compos. Sci. Technol., vol. 56, no. 5, pp. 581-593, Jan. 1996. 


\section{Backup}

4 


\section{Methods to determine knock-down factors (KDF)}
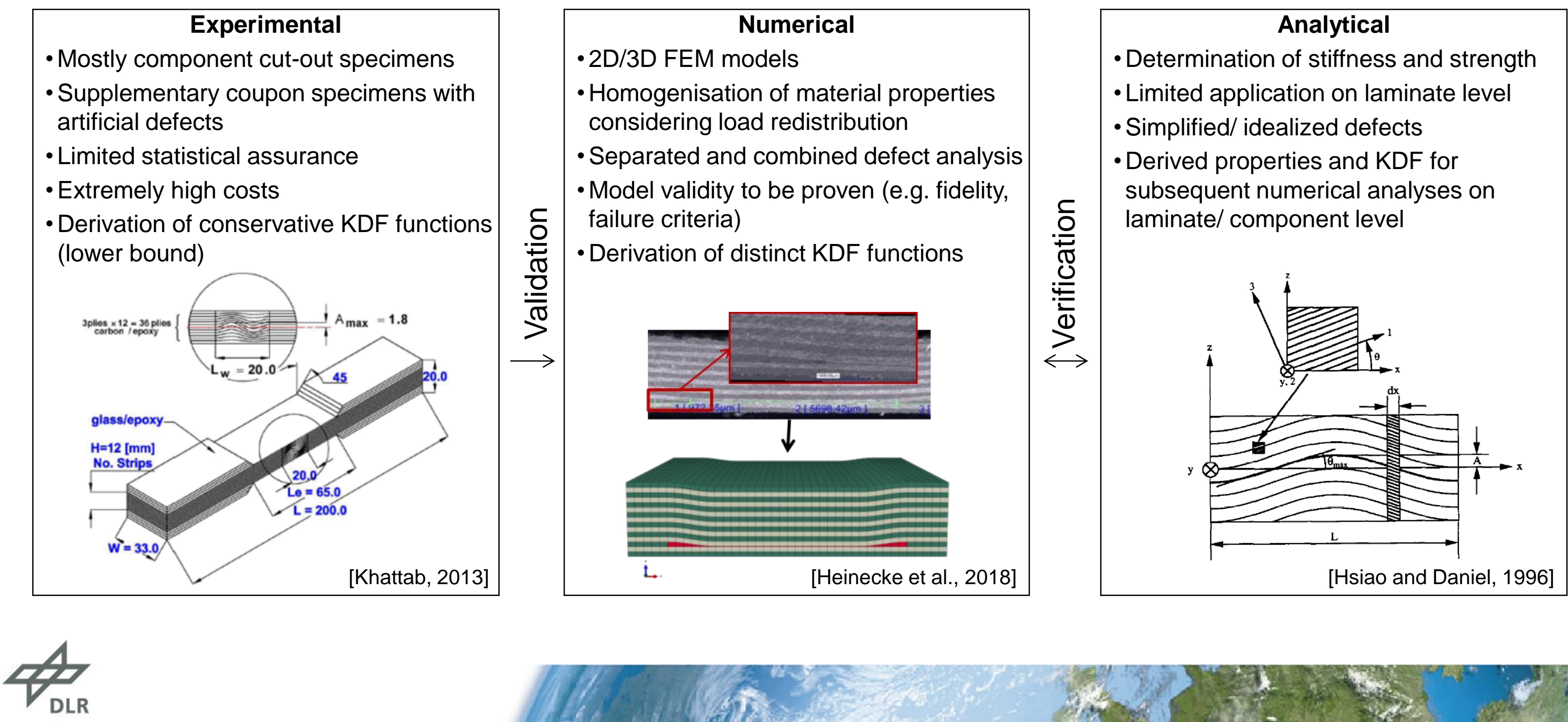


\section{In-situ evaluation of laminates - In a nutshell}

\section{- Demonstration on Wing Cover [Heinecke2018]:}

1. Nominal design and analysis (prior to manufacturing)

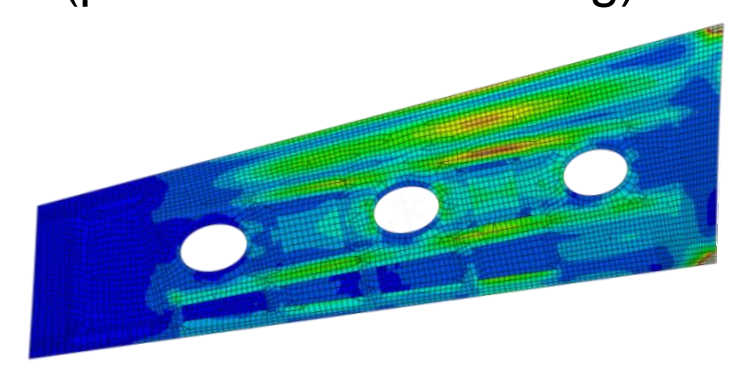

2. AFP manufacturing incl. online measurement

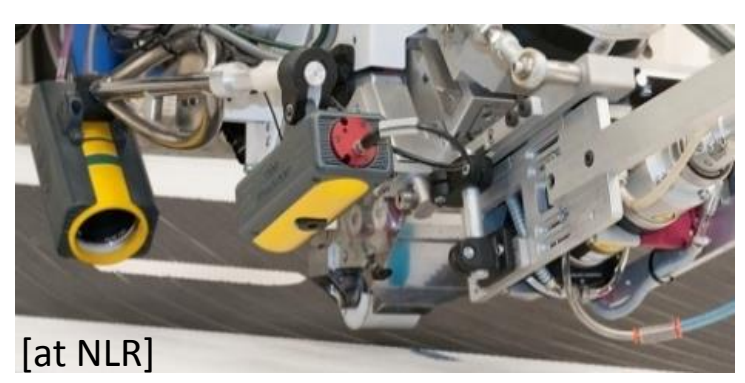

3. In-situ data transfer of defects to manufacturing database

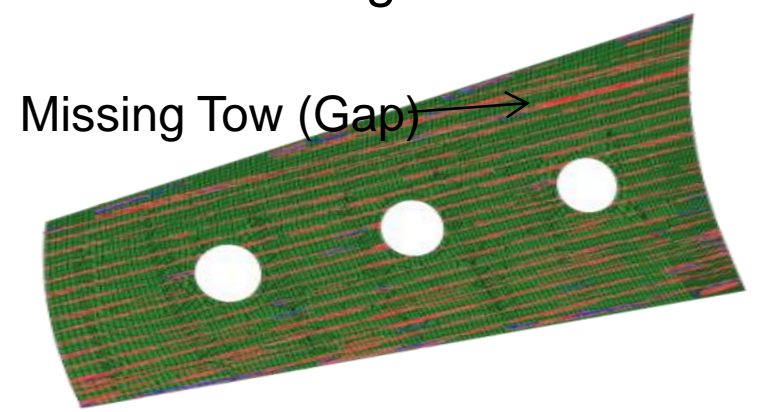

4. In-situ mapping of material properties, model update and structural as-built analysis (re-evaluation)
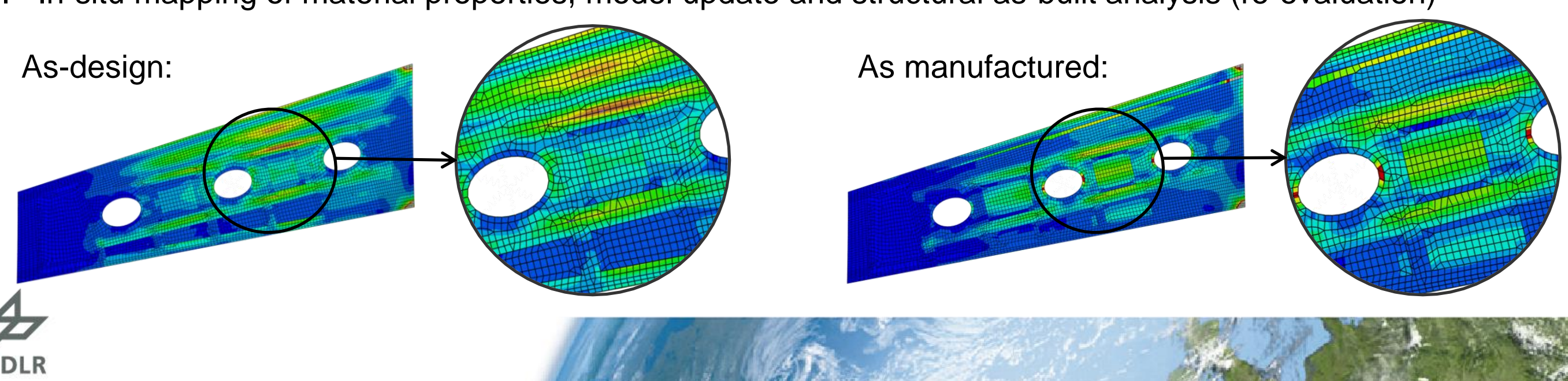

Tsai-Wu

Failure Index

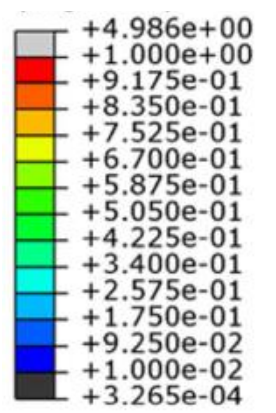

